\title{
A TIME REVERSION OF MARKOV PROCESSES WITH KILLING
}

\author{
By Nobuyuki Ikeda, Masao Nagasawa and Keniti Sato
}

Let $X$ be a conservative Markov process, and $\dot{X}$ be another Markov process obtained from $X$ through killing by $e^{-\varphi} t$, where $\varphi_{t}$ is a continuous non-negative additive functional of $X$. The purpose of this paper is to prove temporally homogeneous Markov property of the reversed process $z_{t}=\dot{x}_{\dot{\xi}-t}$ of $\dot{X}$ from the killing time $\dot{\zeta} .{ }^{1)}$

Hunt proved for Markov chain $x_{\jmath}$ that if $\xi$ is the last exit time from a finite sub-set, then $x_{\xi-\jmath}$ has temporally homogeneous Markov property [1]. This result stimulated us to consider more general reversed processes. The problems of finding appropriate random times from which one can reverse the direction of time and preserve temporally homogeneous Markov property, an extension of this paper, will be discussed in another place by one of the authors. There the proof of Theorem 3. 6 will be simplified from a general point of view.

\section{$\S 1$. Notations and assumptions.}

We use the same terminologies and notations on Markov processes as in [3]. Let $X=\left(x_{t}, \zeta, \mathscr{M}_{t}, \boldsymbol{P}_{a}, \theta_{t}\right)$ be a conservative Markov process satisfying $M_{1} \sim M_{8}$ in [3], and having the strong Feller property. ${ }^{2)}$ The state space of $X$ is denoted by $(E, B)$. Let $\varphi_{t}(\omega)$ be a continuous non-negative additive functional of $X$. We fix $X$ and $\varphi_{t}$ throughout this paper and make two assumptions.

Assumption 1.1. There exist a $\sigma$-finite measure $m$ and a finite measurable function $p(t, a, b)$ of $t>0, a$ and $b \in E$, such that

1) $\boldsymbol{P}_{a}\left[x_{t} \in A\right]=\int_{A} p(t, a, b) m(d b) \quad(t>0, A \in \mathscr{B})$;

2) $p(t, a, b)$ is continuous as a function of $b$;

3) Fixed $t>0, p(t, a, b)$ is bounded as a function of $(a, b)$;

4) Put

Received November 1, 1963.

1) The results of this paper were obtained in joint works at the Sixth Summer Seminar of the Group of Probability and Statistics in 1961.

2) $X$ is said to have the strong Feller property, if $\mathbf{M}_{a}\left[f\left(x_{t}\right)\right] \epsilon C(E)$ for all $f_{\epsilon} B(E)$ and $t>0$. 


$$
g_{\alpha}(a, b)=\int_{0}^{\infty} e^{-\alpha t} p(t, a, b) d t,,^{3)}
$$

then $g_{\alpha}(a, b)$ is, as a function of $a, \alpha$-harmonic in $E \backslash\{b\} \quad(\alpha>0)$.

Assumption 1.2. $\varphi_{t}$ satisfies

$$
\lim _{t \downarrow 0} \sup _{a \in E} \boldsymbol{M}_{a}\left[\varphi_{t}\right]=0,{ }^{4)}
$$

and there exists a $\sigma$-finite measure $n$ such that

$$
\boldsymbol{M}_{a}\left[\int_{0}^{\infty} e^{-\alpha s} d \varphi_{s}\right]=\int_{E} g_{\alpha}(a, b) n(d b)<+\infty \quad(a \in E, \alpha>0) .
$$

Note that Assumption 1.1 implies

$$
\int_{E} p(t, a, b) m(d b) p(s, b, c)=p(t+s, a, c) .
$$

\section{$\S 2$. Transition density of killed process.}

Let $\dot{X}=\left(\dot{x}_{t}, \dot{\zeta}, \dot{g}_{t}, \dot{P}_{a}, \dot{\theta}_{t}\right)$ denote the killed process of $X$ by $e^{-\varphi t}\left(e^{-\varphi_{t}}\right.$-subprocess). We can and shall take $\dot{X}$ satisfying $M_{3}, M_{8}$, and

$$
\dot{\hat{\boldsymbol{P}}}_{a}\left[\dot{x}_{\dot{\zeta}-0} \text { exists } \mid 0<\dot{\zeta}<\infty\right]=1 \text {. }
$$

LeMma 2.1. For each $f \in B(E)$,

$$
\boldsymbol{M}_{a}\left[\int_{0}^{t} \boldsymbol{M}_{x_{s}}\left[f\left(x_{t-s}\right)\right] e^{-\varphi_{s}} d \varphi_{s}\right]=\boldsymbol{M}_{a}\left[\left(1-e^{-\varphi} t\right) f\left(x_{t}\right)\right] .
$$

Proof. Put $\tau_{t}(\omega)=\sup \left\{s ; \varphi_{s}(\omega) \leqq t\right\}$, then $\tau_{t}$ is a Markov time, and we have

$$
\begin{aligned}
& \boldsymbol{M}_{a}\left[\int_{0}^{t} \boldsymbol{M}_{x_{s}}\left[f\left(x_{t-s}\right)\right] e^{-\varphi_{s}} d \varphi_{s}\right] \\
= & \boldsymbol{M}_{a}\left[\left.\int_{0}^{\varphi_{t}} \boldsymbol{M}_{x_{\tau_{s}}}\left[f\left(x_{t-r}\right)\right]\right|_{r=\tau_{s}} e^{-s} d s\right] \\
= & \int_{0}^{\infty} e^{-s} d s \boldsymbol{M}_{a}\left[\chi_{\{s<\varphi t\}} \boldsymbol{M}_{a}\left[f\left(x_{t}\right) \mid \mathcal{M}_{\tau_{s}}\right]\right]
\end{aligned}
$$

3) By 1) and the right continuity of paths, $m$ is positive for any non-empty open set. Hence, $p(t, a, b) \geqq 0$ by virtue of 2$)$, and $g_{x}(a, b)$ is defined $\leqq+\infty$.

4) Put $u(a)=\mathbf{M}_{a}\left[\int_{0}^{\infty} e^{-\alpha s} d \varphi_{s}\right]$. Then $u$ is $\alpha$-excessive and (1.1) is equivalent to the uniform convergence of $\lim _{t \downarrow 0} \mathbf{M}_{a}\left[e^{-\alpha t} u\left(x_{t}\right)\right]=u(\alpha)$. 


$$
\begin{aligned}
& =\int_{0}^{\infty} e^{-s} d s \boldsymbol{M}_{a}\left[\chi_{\left\{s<\varphi_{t}\right\}} f\left(x_{t}\right)\right] \\
& =\boldsymbol{M}_{a}\left[\int_{0}^{\varphi_{t}} e^{-s} d s f\left(x_{t}\right)\right], \text { completing the proof. }
\end{aligned}
$$

Put

$$
q(t, a, b)=p(t, a, b)-\boldsymbol{M}_{a}\left[\int_{0}^{t} p\left(t-s, x_{s}, b\right) e^{-\varphi_{s}} d \varphi_{s}\right]
$$

then $q(t, a, b)$ is the transition density of $\dot{X}$, precisely,

THEOREM 2. 2.

i) $\int_{A} q(t, a, b) m(d b)=\dot{\hat{\boldsymbol{P}}}_{a}\left[\dot{x}_{t} \in A\right]$;

ii) $0 \leqq q(t, a, b) \leqq p(t, a, b)$;

iii) $\int_{E} q(t, a, b) m(d b) q(s, b, c)=q(t+s, a, c)$;

iv) For fixed $(t, b), q(t, a, b)$ is bounded continuous function of $a$;

v) For fixed $(a, b), q(t, a, b)$ is right continuous in $t>0$;

vi) $\dot{X}$ has the strong Feller property.

Proof. i) Let $f=\chi_{A}$, then, using Lemma 2.1, we have

$$
\begin{aligned}
& \int \boldsymbol{M}_{a}\left[\int_{0}^{t} p\left(t-s, x_{s}, b\right) e^{-\varphi_{s}} d \varphi_{s}\right] f(b) m(d b) \\
= & \boldsymbol{M}_{a}\left[\int_{0}^{t} \boldsymbol{M}_{x_{s}}\left[f\left(x_{t-s}\right)\right] e^{-\varphi_{s}} d \varphi_{s}\right]=\boldsymbol{M}_{a}\left[f\left(x_{t}\right)\left(1-e^{-\varphi t}\right)\right] \\
= & \int p(t, a, b) f(b) m(d b)-\dot{\boldsymbol{M}}_{a}\left[f\left(\dot{x}_{t}\right)\right] .
\end{aligned}
$$

ii) $q(t, a, b) \leqq p(t, a, b)$ is obvious. From i), $q(t, a, b) \geqq 0 m$-a.e. $b \in E$. Since Assumption $1.1,2)$ and definition (2. 3) imply that $q(t, a, b)$ is upper semi-continuous in $b$, we have $q(t, a, b) \geqq 0$ for all $b \in E$.

iii)

$$
\int q(t, a, b) m(d b) q(s, b, c)=I_{1}+I_{2}+I_{3}+I_{4}
$$

where 


$$
\begin{aligned}
I_{1} & =\int p(t, a, b) m(d b) p(s, b, c)=p(t+s, a, c), \\
I_{2} & =-\int p(t, a, b) m(d b) \boldsymbol{M}_{b}\left[\int_{0}^{s} p\left(s-r, x_{r}, c\right) e^{-\varphi_{r}} d \varphi_{r}\right] \\
& =-\boldsymbol{M}_{a}\left[\boldsymbol{M}_{x_{t}}\left[\int_{0}^{s} p\left(s-r, x_{r}, c\right) e^{-\varphi_{r}} d \varphi_{r}\right]\right] \\
I_{3} & =-\int \boldsymbol{M}_{a}\left[\int_{0}^{t} p\left(t-u, x_{u}, b\right) e^{-\varphi_{u}} d \varphi_{u}\right] m(d b) p(s, b, c) \\
& =-\boldsymbol{M}_{a}\left[\int_{0}^{t} p\left(t+s-u, x_{u}, c\right) e^{-\varphi_{u}} d \varphi_{u}\right] \\
I_{4} & =\int \boldsymbol{M}_{a}\left[\int_{0}^{t} p\left(t-u, x_{u}, b\right) e^{-\varphi_{u}} d \varphi_{u}\right] m(d b) \boldsymbol{M}_{b}\left[\int_{0}^{s} p\left(s-r, x_{r}, c\right) e^{-\varphi_{r}} d \varphi_{r}\right] \\
& =\boldsymbol{M}_{a}\left[\int_{0}^{t} e^{-\varphi_{u}} d \varphi_{u} \boldsymbol{M}_{x_{t-u}}\left[\int_{0}^{s} p\left(s-r, x_{r}, c\right) e^{-\varphi_{r}} d \varphi_{r}\right]\right] \\
& =\boldsymbol{M}_{a}\left[\left(1-e^{-\varphi_{t}}\right) \boldsymbol{M}_{x_{t}}\left[\int_{0}^{s} p\left(s-r, x_{r}, c\right) e^{-\varphi_{r}} d \varphi_{r}\right]\right],
\end{aligned}
$$

using Lemma 2.1. Finiteness of each term is seen from ii) and (1.3). Therefore, we have

$$
\sum_{i=1}^{4} I_{i}=q(t+s, a, c)
$$

vi) is proved before iv) and v). Put

$$
f_{h}(a)=\boldsymbol{M}_{a}\left[\boldsymbol{M}_{x_{h}}\left[f\left(x_{t-h}\right) e^{-\varphi} t-h\right]\right], \quad \text { for } f \in B(E) .
$$

Then $f_{h}(a)$ is continuous by the strong Feller property of $X$, and

$$
\begin{aligned}
& \left|f_{h}(a)-\boldsymbol{M}_{a}\left[f\left(x_{t}\right) e^{-\varphi} t\right]\right| \\
& =\left|\boldsymbol{M}_{a}\left[\left(1-e^{-\varphi_{h}}\right) \boldsymbol{M}_{x h}\left[f\left(x_{t-h}\right) e^{-\varphi_{t-h}}\right]\right]\right| \\
& \leqq\|f\| \boldsymbol{M}_{a}\left[1-e^{-\varphi_{h}}\right] \leqq\|f\| \boldsymbol{M}_{a}\left[\varphi_{h}\right] \rightarrow 0 \quad(h \downarrow 0),
\end{aligned}
$$

uniformly in $a$ by Assumption 1.2. Hence $\dot{\boldsymbol{M}}_{a}\left[f\left(\dot{x}_{t}\right)\right]$ is continuous, and vi) is obtained.

iv) $q(t, a, b)$ is bounded in $a$ by ii), and from iii)

$$
q(t, a, b)=\dot{M}_{a}\left[q\left(t-t_{0}, \dot{x}_{t_{0}}, b\right)\right] \quad \text { for } \quad 0<t_{0}<t,
$$

which is continuous in $a$ by vi).

v) Noting that $q(t+s, a, b)=\boldsymbol{M}_{a}\left[q\left(t, x_{s}, b\right) e^{-\varphi} s\right]$, right continuity of paths and iv) prove v), completing the proof of Theorem 2.2. 
Put

$$
\dot{g}_{\alpha}(a, b)=\int_{0}^{\infty} e^{-\alpha t} q(t, a, b) d t \quad(\alpha \geqq 0),
$$

then

$$
\dot{\boldsymbol{M}}_{a}\left[\int_{0}^{\infty} e^{-\alpha t} f\left(\dot{x}_{t}\right) d t\right]=\int_{E} \dot{\boldsymbol{g}}_{\alpha}(a, b) f(b) m(d b)
$$

for $f \in B^{+}(E)$.

LEMMA 2. 3.

$$
g_{\alpha}(a, b)=\dot{\boldsymbol{g}}_{\alpha}(a, b)+\boldsymbol{M}_{a}\left[\int_{0}^{\infty} g_{\alpha}\left(x_{s}, b\right) e^{-\alpha s-\varphi_{s}} d \varphi_{s}\right] \quad(\alpha \geqq 0) .
$$

Proof.

$$
\begin{aligned}
& \int_{0}^{\infty} e^{-\alpha t} d t \boldsymbol{M}_{a}\left[\int_{0}^{t} p\left(t-s, x_{s}, b\right) e^{-\varphi_{s}} d \varphi_{s}\right] \\
= & \boldsymbol{M}_{a}\left[\int_{0}^{\infty} e^{-\varphi_{s}} d \varphi_{s} \int_{s}^{\infty} p\left(t-s, x_{s}, b\right) e^{-\alpha t} d t\right] \\
= & \boldsymbol{M}_{a}\left[\int_{0}^{\infty} e^{-\alpha s-\varphi_{s}} d \varphi_{s} \int_{0}^{\infty} p\left(r, x_{s}, b\right) e^{-\alpha r} d r\right] \\
= & \boldsymbol{M}_{a}\left[\int_{0}^{\infty} g_{\alpha}\left(x_{s}, b\right) e^{-\alpha s-\varphi_{s}} d \varphi_{s}\right],
\end{aligned}
$$

which proves (2.5).

LEMmA 2. 4. For each $\alpha>0$ and $f \in B(E)$, we have

$$
\boldsymbol{M}_{a}\left[\int_{0}^{\infty} e^{-\alpha s} f\left(x_{s}\right) d \varphi_{s}\right]=\int_{E} g_{\alpha}(a, b) f(b) n(d b),
$$

and for each $\alpha \geqq 0$ and $f \in B(E)$,

$$
\boldsymbol{M}_{a}\left[\int_{0}^{\infty} e^{-\alpha s-\varphi_{s}} f\left(x_{s}\right) d \varphi_{s}\right]=\int_{E} \dot{\boldsymbol{g}}_{\alpha}(a, b) f(b) n(d b) .
$$

Proof is given in [3] (Theorem 4.1 and 4.2). (2.6) is derived from (1.2), and (2.7) is proved making use of (2.5) and (2.6)

\section{$\S 3$. Time reversion of killed process.}

Definition 3. 1. Put $\Omega^{0}=\left\{\omega ; \dot{\zeta}(\omega)<\infty\right.$, and $\dot{x}_{\dot{\xi}-0}(\omega)$ exists $\}$ and for $\omega \in \Omega^{0}$,

$$
z_{t}(\omega)=\dot{x} \dot{\xi}_{(\omega)-t-0}(\omega), \quad 0 \leqq t<\dot{\zeta}(\omega) .
$$


$z_{t}(\omega)$ is called the reversed process of $\dot{X}$.

In order to prove the temporally homogeneous Markov property of $z_{t}$, we prepare some lemmas.

LEMMA 3.2. If a sequence of finite measures $\mu_{k}(d t)$ on $[0, T](T<\infty)$ converges to a continuous finite measure $\mu(d t)$ in weak, then

$$
\int_{[0, T]} f(t) \mu_{k}(d t) \rightarrow \int_{[0, T]} f(t) \mu(d t) \quad(k \rightarrow \infty),
$$

for each bounded $f$ with at most first kind of discontinuity.

Proof. For $\varepsilon>0$, define a sequence $\left\{t_{\imath}\right\}$ as

$$
\begin{aligned}
t_{0} & =0, \\
t_{\imath+1} & =\inf \left\{t ; t_{i}<t \leqq T,\left|f(t)-f\left(t_{2}\right)\right| \geqq \varepsilon\right\}, \quad(\text { inf } \phi=T),
\end{aligned}
$$

then $t_{i}<t_{\imath+1}$, if $t_{i}<T$, and there exists such $N$ that $t_{N}=T$. Put

$$
f_{\varepsilon}(t)=\sum_{\imath=0}^{N-1} f\left(t_{\imath}\right) \chi_{\left[t_{i}, t_{\imath+1}\right)}(t)+f(T) \chi_{\{T\}}(t),
$$

then $\left|f(t)-f_{\varepsilon}(t)\right|<\varepsilon$. Therefore

$$
\left|\int_{0}^{T} f(t) \mu(d t)-\int_{0}^{T} f_{\varepsilon}(t) \mu(d t)\right| \leqq \int_{0}^{T}\left|f(t)-f_{\varepsilon}(t)\right| \mu(d t)<\varepsilon K,
$$

where $K=\sup _{k} \mu_{k}([0, T])<\infty$.

$$
\begin{aligned}
& \quad\left|\int_{0}^{T} f_{\varepsilon}(t) \mu(d t)-\int_{[0, T]} f_{\epsilon}(t) \mu_{k}(d t)\right| \\
& \leqq \\
& \quad \sum_{i=0}^{N-1}\left|f\left(t_{i}\right)\left\{\mu\left(\left[t_{\imath}, t_{\imath+1}\right)\right)-\mu_{k}\left(\left[t_{i}, t_{\imath+1}\right)\right)\right\}\right| \\
& \quad+\left|f(T)\left\{\mu(\{T\})-\mu_{k}(\{T\})\right\}\right| \rightarrow 0 \quad(k \rightarrow \infty) . \\
& \left|\int_{[0, T]} f_{\varepsilon}(t) \mu_{k}(d t)-\int_{[0, T]} f(t) \mu_{k}(d t)\right|<\varepsilon K .
\end{aligned}
$$

Consequently, combining (3.2), (3.3) and (3.4), we have

$$
\left|\int_{0}^{T} f(t) \mu(d t)-\int_{[0, T]} f(t) \mu_{k}(d t)\right|<3 \varepsilon K,
$$

for sufficiently large $k$, completing the proof.

LEMMA 3. 3. For $k \geqq 0, f_{0}, f_{1}, \cdots, f_{k} \in B(E)$, and $0=t_{0} \ll t_{1}<\cdots<t_{k}$, we have

$$
\dot{\boldsymbol{M}}_{a}\left[\prod_{\jmath=0}^{k} f_{j}\left(z_{t_{j}}\right) ; t_{k}<\dot{\zeta}<\infty\right]=\boldsymbol{M}_{a}\left[\int_{t_{k}}^{\infty} \prod_{j=0}^{k} f_{j}\left(x_{t-t_{j}}\right) e^{-\varphi_{t}} d \varphi_{t}\right] .
$$


Proof. It suffices to prove (3.5) for $f_{0}, f_{1}, \cdots, f_{k} \in C(E)$. Since the discontinuity points of $x_{t}(\omega)$ are at most countable, we have

$$
\begin{aligned}
& \boldsymbol{M}_{a}\left[\int_{t_{k}}^{\infty} I_{\jmath=0}^{k} f_{j}\left(x_{t-t_{j}}\right) e^{-\varphi t} d \varphi_{t}\right]=\boldsymbol{M}_{a}\left[\int_{t_{k}}^{\infty} \prod_{\jmath=0}^{k} f_{j}\left(x_{t-t_{j}-0}\right) e^{-\varphi t} d \varphi_{t}\right] \\
& =\lim _{h \downarrow 0} \sum_{i=0}^{\infty} \boldsymbol{M}_{a}\left[\prod_{j=0}^{k} f_{j}\left(x_{t_{k^{+}}+i h-t_{j}}\right)\left(e^{-\varphi_{t_{k}}+i n}-e^{\left.-\varphi_{t_{k}+(i+1) h}\right)}\right]\right. \\
& =\lim _{h \downarrow 0} \sum_{i=0}^{\infty} \dot{\boldsymbol{M}}_{a}\left[\prod_{j=0}^{k} f_{j}\left(\dot{x}_{t_{k}+i h-t_{j}}\right) ; t_{k}+i h<\dot{\zeta} \leqq t_{k}+(i+1) h\right] \\
& =\dot{\boldsymbol{M}}_{a}\left[\prod_{\jmath=0}^{k} f_{j}\left(\dot{x}_{\xi_{-} t_{j}-0}\right) ; t_{k}<\dot{\zeta}<\infty\right] \\
& =\dot{\boldsymbol{M}}_{a}\left[\prod_{j=0}^{k} f_{j}\left(z_{t_{j}}\right) ; t_{k}<\dot{\zeta}<\infty\right] \text {. }
\end{aligned}
$$

Here we made use of conservativity of $X$.

The next theorem is a generalization of Lemma 2.4,

Theorem 3. 4. For $k \geqq 1, f_{0}, f_{1}, \cdots, f_{k} \in B(E)$, and $0=t_{0}<t_{1}<\cdots<t_{k}$, and $\alpha \geqq 0$, we have

$$
\boldsymbol{M}_{a}\left[\int_{t_{k}}^{\infty} \prod_{\jmath=0}^{k} f_{j}\left(x_{t-t_{j}}\right) e^{-\alpha t-\varphi t} d \varphi_{t}\right]
$$

(3.6) $=e^{-\alpha t_{k}} \int_{E} \ldots \int_{E} n\left(d a_{0}\right) f_{0}\left(a_{0}\right) q\left(t_{1}, a_{1}, a_{0}\right) m\left(d a_{1}\right) f_{1}\left(a_{1}\right) q\left(t_{2}-t_{1}, a_{2}, a_{1}\right) m\left(d a_{2}\right) f_{2}\left(a_{2}\right)$

$$
\cdots q\left(t_{k}-t_{k-1}, a_{k}, a_{k-1}\right) m\left(d a_{k}\right) f_{k}\left(a_{k}\right) \dot{\boldsymbol{g}}_{\alpha}\left(a, a_{k}\right)
$$

Proof. It is sufficient to prove (3.6) for $f_{0}, f_{1}, \cdots, f_{k} \in C_{0}^{+}(E)$, and $\alpha>0$. The case $\alpha=0$ is obtained by approximation of $\alpha$. Put

$$
u(a)=\boldsymbol{M}_{a}\left[\int_{0}^{\infty} e^{-\alpha_{0} t} d \varphi_{t}\right] \text { and } v_{N}(a)=N\left\{u(a)-e^{-\alpha_{0} / N} \boldsymbol{M}_{a}\left[u\left(x_{1 / N}\right)\right]\right\}
$$

for some $\alpha_{0}>0$. Then

$$
\boldsymbol{P}_{a}\left[\int_{0}^{t} e^{-\alpha_{0} s} v_{N}\left(x_{s}\right) d s \rightarrow \int_{0}^{t} e^{-\alpha_{0} s} d \varphi_{s}, N \rightarrow \infty, \text { for all } t\right]=1,
$$

(cf. [2] and [4]). For $\beta>0$, on account of Lemma 3. 2, we have

$$
\int_{t_{k-1}}^{\infty} e^{-\beta t_{k}} d t_{k} \boldsymbol{M}_{a}\left[\int_{t_{k}}^{\infty} \prod_{\jmath=0}^{k} f_{j}\left(x_{t-t_{j}}\right) e^{-\alpha t-\varphi t} d \varphi_{t}\right]
$$




$$
\begin{aligned}
& =\lim _{T \rightarrow \infty} \lim _{N \rightarrow \infty} \int_{t_{k-1}}^{T} e^{-\beta t_{k}} d t_{k} \boldsymbol{M}_{a}\left[\int_{t_{k}}^{T} \prod_{j=0}^{k} f_{j}\left(x_{t-t_{j}}\right) e^{-\alpha t-\varphi t} v_{N}\left(x_{t}\right) d t\right] \\
& =\lim _{T^{\prime} \rightarrow \infty} \lim _{N \rightarrow \infty} \int_{t_{k-1}}^{T} e^{-\beta t_{k}-\alpha t_{k}} d t_{k} \boldsymbol{M}_{a}\left[\int_{0}^{T-t_{k}} \prod_{j=0}^{k} f_{j}\left(x_{t_{k}+t-t_{j}}\right) e^{-\alpha t-\varphi_{t}+t_{k}} v_{N}\left(x_{t_{k}+t}\right) d t\right] \\
& =\lim _{T \rightarrow \infty} \lim _{N \rightarrow \infty} \int_{0}^{T-t_{k-1}} e^{-\alpha t} d t \boldsymbol{M}_{a}\left[f_{k}\left(x_{\imath}\right) e^{-\varphi t} \boldsymbol{M}_{x_{t}}\left[\int_{t_{k}-1}^{T-t} e^{-\beta t_{k}-\alpha t_{k}} d t_{k} \prod_{j=0}^{k-1} f_{j}\left(x_{t_{k}-t j}\right) v_{N}\left(x_{t_{k}}\right) e^{-\varphi t_{k}}\right]\right] \\
& =\int_{0}^{\infty} e^{-\alpha t} d t \boldsymbol{M}_{a}\left[f_{k}\left(x_{t}\right) e^{-\varphi t} \boldsymbol{M}_{x_{t}}\left[\int_{t_{k-1}}^{\infty} e^{-\beta t_{k}-\alpha t_{k}-\varphi t_{k}} \prod_{j=0}^{k-1} f_{j}\left(x_{t_{k}-t_{j}}\right) d \varphi_{t_{k}}\right]\right] .
\end{aligned}
$$

This is, if (3.6) is valid for $k-1$,

$$
=\int_{t_{k-1}}^{\infty} e^{-\beta t_{k}} d t_{k} e^{-\alpha t_{k}} \int \cdots \int n\left(d a_{0}\right) f_{0}\left(a_{0}\right) q\left(t_{1}, a_{1}, a_{0}\right) \cdots q\left(t_{k}-t_{k-1}, a_{k}, a_{k-1}\right) m\left(d a_{k}\right) f_{k}\left(a_{k}\right) \dot{\boldsymbol{g}}_{\alpha}\left(a, a_{k}\right) .
$$

The right member of (3.6) is right continuous in $t_{k}$ by Assumption 1.1 and the left member of (3.6) is also right continuous in $t_{k}$ by the following calculation. Consequently, we have (3.6) by stripping off the Laplace transform. If $k=1,(2.7)$ and similar argument lead to (3.6). Let $h \downarrow 0$.

$$
\begin{aligned}
& \left|\boldsymbol{M}_{a}\left[\int_{t_{k}+h}^{\infty} \prod_{\jmath=0}^{k-1} f_{j}\left(x_{t-t_{j}}\right) f_{k}\left(x_{t-t_{k}-h}\right) e^{-\alpha t-\varphi t} d \varphi_{t}\right]-\boldsymbol{M}_{a}\left[\int_{t_{k}}^{\infty} \prod_{\jmath=0}^{k-1} f_{j}\left(x_{t-t_{j}}\right) f_{k}\left(x_{t-t_{k}}\right) e^{-\alpha t-\varphi t} d \varphi_{t}\right]\right| \\
& \leqq \boldsymbol{M}_{a}\left[\int_{t_{k}+h}^{\infty} \prod_{\jmath=0}^{k-1} f_{j}\left(x_{t-t_{j}}\right)\left|f_{k}\left(x_{t-t_{k}-h}\right)-f_{k}\left(x_{t-t_{k}}\right)\right| e^{-\alpha t-\varphi t} d \varphi_{\iota}\right] \\
& \quad+\boldsymbol{M}_{a}\left[\int_{t_{k}}^{t_{k}+h} \prod_{\jmath=0}^{k} f_{j}\left(x_{t-t_{j}}\right) e^{-\alpha t-\varphi_{t}} d \varphi_{t}\right] \\
& \rightarrow 0
\end{aligned}
$$

because $x_{t-t_{k}-h}(\omega) \rightarrow x_{t-t_{k}}(\omega)(h \downarrow 0)$ except countable values of $t$, completing the proof.

Let $\nu$ be a $\sigma$-finite measure on $E$, and put

$$
\begin{aligned}
\eta(a) & =\int_{E} \nu(d b) \dot{g}_{0}(b, a), \\
E_{\eta} & =\{a ; a \in E, 0<\eta(a)<\infty\} \text { and } E_{0}=E \backslash E_{\eta} .
\end{aligned}
$$

Lemma 3.5. If $\nu$ satisfies $\dot{\boldsymbol{P}}_{\nu}\left[z_{t} \in K\right]<+\infty$ for every compact $K \subset E{ }^{5)}$ then

$$
\dot{\boldsymbol{P}}_{\nu}\left[z_{t} \in E_{0}\right]=0 \quad(t \geqq 0) .
$$

Proof. Choose a sequence of compact sets $K_{3} \uparrow E$. By Theorem 3. 4,

5) $\dot{\boldsymbol{P}}_{\nu}[B]=\int \nu(d a) \dot{\boldsymbol{P}}_{a}[B]$ 


$$
\dot{\boldsymbol{P}}_{\nu}\left[z_{t} \in E_{0} \frown K_{j}\right]=\iint n(d a) q(t, b, a) m(d b) \chi_{E_{0} \frown \boldsymbol{K}_{j}}(b) \eta(b) .
$$

The right side of the above is zero or infinity, but the left side is finite by the assumption. Therefore $\dot{\boldsymbol{P}}_{\nu}\left[z_{t} \in E_{0} \frown K_{j}\right]=0$. Letting $j \rightarrow \infty$, we have (3. 10).

Theorem 3.6. Let $\nu$ be an initial measure of $X$ satisfying $\dot{\boldsymbol{P}}_{\nu}\left[z_{t} \in K\right]<+\infty$ for every compact $K \subset E$. Then the reversed process $\left(z_{t}, \dot{\boldsymbol{P}}_{\nu}\right)$ of $\left(\dot{x}_{t}, \dot{\boldsymbol{P}}_{\nu}\right)$ has temporally homogeneous Markov property and

$$
\dot{\boldsymbol{P}}_{\nu}\left[z_{t} \in d b \mid z_{s}=a\right]=q(t-s, b, a) \frac{\eta(b)}{\eta(a)} m(d b) \quad(0 \leqq s<t) .
$$

The initial measure of the reversed process is

$$
\dot{\boldsymbol{P}}_{\nu}\left[z_{0} \in d a\right]=\eta(a) n(d a) .
$$

Here $\eta(a)$ is defined by (3.8).

Proof. For $f_{0}, f_{1}, \cdots, f_{k} \in B(E)$ and $0=t_{0}<\cdots<t_{k}$, we have

$$
\begin{aligned}
& \dot{\boldsymbol{M}}_{\nu}\left[\prod_{j=0}^{k} f_{j}\left(z_{t_{j}}\right) ; t_{k}<\dot{\zeta}\right] \\
& =\dot{\boldsymbol{M}}_{\nu}\left[\prod_{j=0}^{k} f_{j}\left(z_{t_{j}}\right) \chi_{E_{\eta}}\left(z_{t_{k-1}}\right) ; t_{k}<\dot{\zeta}\right] \\
& =\int \cdots \int n\left(d a_{0}\right) f_{0}\left(a_{0}\right) q\left(t_{1}, a_{1}, a_{0}\right) m\left(d a_{1}\right) f_{1}\left(a_{1}\right) \cdots \\
& \cdots m\left(d a_{k-1}\right) f_{k-1}\left(a_{k-1}\right) \eta\left(a_{k-1}\right) \chi_{E_{\eta}}\left(a_{k-1}\right) \int q\left(t_{k}-t_{k-1}, a_{k}, a_{k-1}\right) \frac{\eta\left(a_{k}\right)}{\eta\left(a_{k-1}\right)} f_{k}\left(a_{k}\right) m\left(d a_{k}\right) \\
& =\dot{\boldsymbol{M}}_{\nu}\left[\prod_{j=0}^{k-1} f_{j}\left(z_{t_{j}}\right) \chi_{E_{\eta}}\left(z_{t_{k-1}}\right) \int q\left(t_{k}-t_{k-1}, a_{k}, z_{t_{k-1}}\right) \frac{\eta\left(a_{k}\right)}{\eta\left(z_{t_{k-1}}\right)} f_{k}\left(a_{k}\right) m\left(d a_{k}\right) ; t_{k-1}<\dot{\zeta}\right] \text {, }
\end{aligned}
$$

by Lemma 3.3, Theorem 3. 4 and Lemma 3.5. This proves the temporally homogeneous Markov property of $\left(z_{t}, \dot{\boldsymbol{P}}_{\nu}\right)$ together with (3.12). (3.13) follows directly from (2.7) and Lemma 3. 3.

REMARK 3. 7. Suppose that $m$ is a sub-invariant measure for $X$. Then, as shown in [3],

$$
\int_{E} n(d b) \dot{\boldsymbol{g}}_{0}(b, a)=1 \quad \text { for } \quad(m+n) \text {-a.e. } \quad a \in E
$$

holds under some additional conditions. Thus, in this case, the reversed process $\left(z_{t}, \dot{\boldsymbol{P}}_{n}\right)$ of $\left(\dot{x}_{t}, \dot{\boldsymbol{P}}_{n}\right)$ has transition density $q(t, b, a) m(d b)$ and the initial measure $n(d b)$. This gives a probabilistic interpretation of adjoint processes. 
REMARK 3. 8. Under the assumption of $\S 6$ of [3], we are able to prove a theorem of reversed processes analogous to Theorem 3.6. In this case, Theorem is stated as: Under the notations of $\S 6$ of [3], the reversed process $\left(z_{t}, \dot{\boldsymbol{P}}_{\nu}\right)$ of $\left(\dot{x}_{t}, \dot{\boldsymbol{P}}_{\nu}\right)$ is a version of $\left(\dot{\hat{\boldsymbol{x}}}_{t}, \dot{\hat{\boldsymbol{P}}}_{\eta n}^{\eta}\right)$, where $\dot{\hat{X}}^{\eta}=\left(\dot{\hat{\boldsymbol{x}}}_{t} . \hat{\hat{\boldsymbol{P}}}_{a}^{\eta}\right)$ is the super-harmonic transform of $\hat{X}=\left(\dot{\hat{\boldsymbol{x}}}_{t}, \dot{\hat{\boldsymbol{P}}}_{a}\right)$ by $\eta$.

\section{REFERENCES}

[1] Hunt, G. A., Markoff chains and Martin boundaries. Ill. J. Math. 4 (1960), $316-340$.

[2] Ineda, N., K. Sato, H. Tanaka, and T. Ueno, Boundary problems in multidimensional diffusion processes II. Seminar on Prob. 6 (1961), 1-131. (Japanese)

[3] Nagasawa, M., and K. Sato, Some theorems on time change and killing of Markov processes. Kōdai Math. Sem. Rep. 15 (1963), 195-219.

[4] VolkonskiI, V. A., Additive functionals of Markov processes. Trudy Mosk. Mat. Ob. 9 (1960), 143-190. (Russian)

Department of Mathematics, Osaka University, Mathematical Institute, Nagoya University, and

Department of Mathematics, Tokyo Metropolitan University. 